\title{
Decreased bone mineral density and alteration in biochemical bone metabolism markers in children affected by bone tumors after completion of therapy
}

\author{
J. AMBROSZKIEWICZ1,*, J. GAJEWSKA ${ }^{1}$, E. ROGOWSKA ${ }^{2}$, K. SZAMOTULSKA ${ }^{3}$, M. CHELCHOWSKA ${ }^{1}$, G. ROWICKA ${ }^{4}$, \\ M. RYCHLOWSKA-PRUSZYNSKA ${ }^{2}$
}

${ }^{1}$ Screening Department; ${ }^{2}$ Department of Oncological Surgery for Children and Youth; ${ }^{3}$ Department of Epidemiology; ${ }^{4}$ Department of Nutrition, Institute of Mother and Child, Warsaw, Poland

*Correspondence: jagoda.ambroszkiewicz@imid.med.pl

Received April 26, 2014 / Accepted July 8, 2014

\begin{abstract}
The aim of this study was to assess bone mineral density (BMD) and biochemical bone metabolism markers in patients with bone tumors after anti-cancer treatment.

The study included 27 patients (median age 15 years) with malignant bone tumors and 27 healthy children. In all subjects, $\mathrm{BMD}$ and body composition were measured by dual-energy X-ray absorptiometry. Serum bone markers were determined by immunoenzymatic assays. After completion of treatment, patients with bone tumors had significantly decreased total and lumbar spine BMD. We observed lower calcium and vitamin D levels in patients and comparable values of bone turnover markers (carboxyterminal telopeptide of collagen type I - CTX, bone alkaline phosphatase - BALP and osteocalcin - OC) in both groups of children. However, the level of carboxylated osteocalcin (cOC) was significantly lower $(\mathrm{p}<0.01)$ and undercarboxylated OC (ucOC) was higher $(\mathrm{p}<0.05)$ in patients than in the controls. Additionally, we observed similar values of anthropometric parameters in the subgroups of patients treated with methotrexate (MTX) or without MTX. In patients treated without MTX we found lower $(\mathrm{p}<0.05)$ ratio of $\mathrm{COC} / \mathrm{ucOC}$, lower vitamin D level and higher CTX concentration.

Patients with bone tumors after anticancer treatment had decreased bone mineral density and alterations in bone metabolism markers with potential decrease in bone formation.
\end{abstract}

Key words: bone cancer survivors, bone mineral density, bone formation markers, bone resorption markers, methotrexate

One of the most difficult problems in pediatric oncology is the diagnosis and treatment of malignant bone tumors, which often appear in the second decade of life. Among them osteosarcoma, Ewing's sarcoma and primitive neuroectodermal tumor (PNET) are the most common sarcomatous bone tumors in young patients. Improvement of comprehensive cancer treatment consisting of a combination of chemotherapy (preoperative and postoperative), radiotherapy and surgery enables long-term survivors. The 5-year survival rate for non-metastatic bone cancers increased to about $60-70 \%$ in recent years, however, metastatic or multifocal tumors are still a severe problem [1-2]. The survivors are at increased risk for a variety of health problems resulting from cancer or its treatment. One such problem is altered bone metabolism that may interfere with the attainment of the appropriate peak bone mass. Lower bone mass predisposes patients with bone cancers to a higher risk of osteopenia, osteoporosis, and bone fractures. Most of the existing literature reports reduced total and regional bone mineral density (BMD) values in adult longterm bone sarcoma survivors but only a few studies concerning children and adolescents have been conducted [3-7].

The etiology of BMD deficit in pediatric oncologic patients is multifactor and includes both direct effects of the tumor and its treatment. One of the standard agents of osteosarcoma treatment is methotrexate (MTX). The main mechanism of action of MTX is inhibition of dihydrofolate reductase, which reduces folate to tetrahydrofolate, an essential cofactor in RNA and DNA synthesis [8-9]. High doses of MTX have been known to be highly cytotoxic to bone cells and affect bone remodeling by inhibiting the differentiation of osteoblasts in the early stage [10-11]. However, clinical studies conducted in patients with bone cancers showed different results. Some 
researchers reported similar values but others found decreased bone mineral density in patients treated with chemotherapy with and without MTX $[5,12]$. It is unclear whether low BMD in patients with malignant bone tumors is related to high-doses of MTX or other factors.

Apart from measuring BMD, determinations of biochemical bone metabolism markers give information on global metabolic bone status and can detect subtle alterations in the skeleton over short periods of time. The balance between bone formation and resorption processes is controlled by the complex of cytokines such as osteoprotegerin (OPG), receptor activator of nuclear factor $\kappa \mathrm{B}$ (RANK), and receptor activator of nuclear factor $\kappa B$ ligand (RANKL) [13-14]. There is scarce information about levels of bone metabolism markers in patients with bone tumors after anti-cancer treatment. The authors observed normal, reduced and elevated serum concentrations of bone markers in osteosarcoma and Ewing `s sarcoma survivors $[4,5,15]$. To date no study to assess the RANK/RANKL/OPG system or its modulation by MTX in children and adolescents with bone cancers after therapy has been conducted.

Therefore, the aim of this study was to assess bone mineral density and biochemical bone metabolism markers in patients with malignant bone tumors after anti-cancer treatment.

\section{Patients and methods}

Patients. The study included 27 patients (12 girls, 15 boys; median age 15 years, range 7-18 years) with malignant bone tumors treated at the Department of Surgical Oncology for Children and Youth, Institute of Mother and Child in Warsaw. The clinical characteristics of these patients are summarized in Table 1. Only those patients who had received standard treatment, which consisted of preoperative chemotherapy, followed by resection of the tumor, and postoperative chemotherapy were included in this study. Among them, there were $17 \mathrm{pa}$ tients with osteosarcoma, 8 patients with Ewing `s sarcoma, and 2 patients with PNET. In most subjects, the primary tumor was localized in the femur and the tibia. Patients with osteosarcoma received preoperative chemotherapy (according to EURAMOS) composed of high-dose methotrexate (with leucovorin rescue), cisplatin, and doxorubicin. After surgery, histological "good responders" (showing $>90 \%$ or more tumor necrosis) received the same type of chemotherapy, whereas "poor responders" (less than $90 \%$ of tumor necrosis) had their chemotherapeutic protocol changed to varying combinations of ifosfamide-, etoposide- and VP16-added chemotherapy (according to MAPIE protocol). Among patients affected by osteosarcoma, the histological response of the primary tumor was good in $35 \%$ of the cases and poor in $65 \%$. The group of patients with Ewing 's sarcoma and PNET (70\% with metastases and $30 \%$ without metastases) received chemotherapy according to Euro-Ewing 99 protocol (neoadjuvant - according to VIDE: vincristine, ifosfamide, doxorubicin, etoposide and adjuvant - according to VAI or VAC: vincristine, actinomycin-D, ifosfamide or cyclophosphamide). Three of the patients with Ewing's sarcoma received also local radiotherapy, because of partially bad response to chemotherapy or anatomically problematic wide resection. The average duration of treatment in the whole group of patients was $12.6 \pm 2.9$ months (range 7.519.0), in the subgroup of patients treated with MTX: 11.2 \pm 2.1 months (range 7.5-15.5), in the subgroups of patients treated without MTX: $15.5 \pm 2.1$ months (range 13.0-19.0) $(\mathrm{p}<0.001$ between two subgroups of patients).

The control group consisted of 27 healthy subjects matched for age and gender (12 girls, 15 boys; median age 14 years, range 7-18 years) free from diseases affecting growth rate and bone metabolism. This study protocol was approved by the Ethics Committee of the Institute of Mother and Child and informed consent was obtained from study participants or their parents. The investigation conforms to the principles outlined in the Declaration of Helsinki.

Methods. The anthropometric data (height, weight, body mass index) were measured at the time of densitometry determination in patients with bone tumors after completion of treatment (exactly one month after the last postoperative chemotherapy course) and in healthy subjects. Bone mineral density of total body (tBMD), BMD of the lumbar spine (BMD L2-L4), as well as body composition details (bone mineral content - BMC, fat mass, lean mass) were performed by dual-energy X-ray absorptiometry (DXA) using the Lunar Prodigy system (General Electric Healthcare, UK) with the pediatric software 9.30.044. The measurements were performed using standard positioning techniques on the same machine in all the studied subjects. The data were compared with that of the reference population and the results expressed as Z-scores.

For biochemical measurements, blood was taken in the morning after overnight fasting, and serum samples were

Table 1. Clinical characteristics of patients with malignant bone tumors

\begin{tabular}{llcc}
\hline \multirow{2}{*}{ Gender } & Male & $\begin{array}{c}\text { Number of } \\
\text { patients }\end{array}$ & $\begin{array}{c}\text { Percent of } \\
\text { patients }\end{array}$ \\
\hline \multirow{3}{*}{ Type of tumor } & Female & 15 & $56 \%$ \\
& Osteosarcoma & 12 & $44 \%$ \\
Localization of primary & 17 & $63 \%$ \\
tumor & Ewing's sarcoma & 8 & $30 \%$ \\
& PNET & 2 & $7 \%$ \\
& Tibia & 12 & $44.5 \%$ \\
& Humerus & 6 & $22 \%$ \\
Chemotherapy regiments & Fibula & 3 & $11 \%$ \\
& Other & 2 & $7.5 \%$ \\
& With MTX & 4 & $15 \%$ \\
Metastasis & Without MTX & 10 & $63 \%$ \\
& With metastases & 18 & $37 \%$ \\
Type of surgery & Without metastases & 9 & $67 \%$ \\
& Resection & 19 & $70 \%$ \\
\hline
\end{tabular}

MTX - methotrexate 
obtained after centrifugation (1000xg, $10 \mathrm{~min}$ at $\left.4^{\circ} \mathrm{C}\right)$. Serum levels of calcium, phosphate and total alkaline phosphatase (ALP) were analyzed by standard laboratory methods on an automatic analyzer the same morning. The remaining serum samples were stored at $-70^{\circ} \mathrm{C}$ for later analyses of 25-hydroxyvitamin D (kits from DiaSorin, USA) and bone metabolism markers (immunoenzymatic ELISA assays). To evaluate osteocalcin (OC) and carboxyterminal telopeptide of collagen type I (CTX) levels, we used kits from IDS (UK), bone alkaline phosphatase (BALP) and osteoprotegerin (OPG) from Quidel (USA), soluble receptor activator of nuclear factor kap$\mathrm{paB}$ ligand (sRANKL) from ImmunoDiagnostic (Germany), carboxylated ( $\mathrm{COC}$ ) and undercarboxylated (ucOC) forms of osteocalcin - kits from Takara (Japan). The intra- and interassay coefficients of variation $(\mathrm{CVs})$ of these methods were less than $10 \%$.

Statistical analysis. All variables were tested for normality using the Kolmogorov-Smirnov test with Lilliefors significance correction. Data are presented as means \pm SD (normal distribution) or median and 25-75 percentiles (skewed distribution). Group differences were determined by paired t-Student or Wilcoxon tests as appropriate. Additionally, Spearman rank correlations were calculated. First, the data were analyzed comparing all cancer cases $(n=27)$ with the controls $(n=27)$. Then patients were divided into two subgroups stratified by using MTX-added chemotherapy $(\mathrm{n}=17$ patients) and chemotherapy without MTX $(\mathrm{n}=10)$. The patient subgroups were compared with healthy subjects matched for age and gender. The data were presented as differences between patients and controls. For comparisons of the magnitude of differences between cases and matched controls by type of chemotherapy (MTX and non-MTX), the Mann-Whitney exact test was used. The significance level was set at $\mathrm{p}<0.05$. For all statistical calculations we used IBM-SPSS v.18.0 software.

\section{Results}

The two groups of studied children and adolescents were similar according to weight, height and body mass index (Table 2). Patients with bone tumor after chemotherapy had significantly higher fat mass $(\mathrm{p}<0.01)$ and lower lean mass $(\mathrm{p}<0.05)$ than healthy children. Survivors had also lower total body BMC compared with controls $(p<0.01)$. We observed significantly lower mean value of total $\mathrm{BMD}(\mathrm{p}<0.05)$ and lumbar spine BMD L2-L4 $(\mathrm{p}<0.05)$ as well as their Z-scores $(\mathrm{p}<0.01)$ in the patient group than in the healthy children. In the whole group of patients with bone tumor, $61 \%$ had Z-score of total body BMD between -1 and 1, 19.5\% between -1 and -2 , and $19.5 \%$ lower than -2 . Additionally, $48 \%$ of our patients had Z-score BMD L2-L4 between 1 and -1, 38\% between -1 and -2 and $12 \%$ lower than -2 . We observed strong and statistically significant positive correlations between body weight, body height, BMI, fat mass, lean mass, BMC and BMDs in the whole group of patients (all Spearman 's rhos within range of $0.5-0.9$, all $p$ values $<0.01$ ).

In the whole group of patients after completion of antitumor treatment, we observed decreased concentration of serum phosphate $(\mathrm{p}<0.05)$, calcium $(\mathrm{p}<0.05)$, and 25 -hydroxyvitamin $\mathrm{D}(\mathrm{p}<0.001)$ in comparison with healthy children (Table 3). In details, we observed vitamin D deficiency (less than $20 \mathrm{ng} / \mathrm{ml}$ ) in $78 \%$ and suboptimal vitamin D status (20$30 \mathrm{ng} / \mathrm{ml}$ ) in $22 \%$ of the cancer survivors. On the contrary, in healthy children $44 \%$ of the subjects had vitamin D deficiency and $56 \%$ had concentrations of this vitamin in the range of 20-30 ng/ml.

Regarding bone turnover markers, we observed comparable serum values of bone formation markers (ALP, BALP, total osteocalcin) in both groups of children. However, the concentration of carboxylated form of osteocalcin was significantly $(\mathrm{p}<0.01)$ lower and undercarboxylated OC was higher

Table 2. Values of anthropometric parameters in the two studied groups of children

\begin{tabular}{lccc}
\hline & Bone tumor survivors & Healthy children & p \\
\hline Number & 27 & 27 & $14.0(12.5-16.0)$ \\
Age $(\text { years })^{\mathrm{b}}$ & $15.0(12.0-16.0)$ & $160.9 \pm 17.2$ & 0.090 \\
Height $(\mathrm{cm})^{\mathrm{a}}$ & $159.8 \pm 13.8$ & $51.5 \pm 15.5$ & 0.354 \\
Weight $(\mathrm{kg})^{\mathrm{b}}$ & $53.8 \pm 17.0$ & $18.9(17.3-20.6)$ & 0.419 \\
Body mass index $\left(\mathrm{kg} / \mathrm{m}^{2}\right)^{\mathrm{b}}$ & $20.5(18.1-22.9)$ & $11.9(6.6-16.5)$ & 0.137 \\
Total fat mass $(\mathrm{kg})^{\mathrm{b}}$ & $16.0(10.9-22.5)$ & $37.1 \pm 11.9$ & 0.009 \\
Total lean mass $(\mathrm{kg})^{\mathrm{a}}$ & $32.3 \pm 9.2$ & $2048.1 \pm 757.3$ & 0.011 \\
Total body BMC $(\mathrm{g})^{\mathrm{a}}$ & $1711.1 \pm 576.4$ & $1.028 \pm 0.141$ & 0.008 \\
Total body BMD $\left(\mathrm{g} / \mathrm{cm}^{2}\right)^{\mathrm{a}}$ & $0.960 \pm 0.136$ & $0.109 \pm 1.059$ & 0.019 \\
Total body BMD Z-score & $-1.164 \pm 1.118$ & $0.974 \pm 0.216$ & 0.001 \\
Lumbar spine BMDL2-L4 (g/cm $)^{\mathrm{a}}$ & $0.922 \pm 0.216$ & $-0.096 \pm 1.039$ & 0.032 \\
Lumbar spine BMDL2-L4 Z-score $^{\mathrm{a}}$ & $-1.059 \pm 1.048$ & 0.002 \\
\hline
\end{tabular}

Data are presented as ${ }^{a}$ mean values \pm SD (paired t-Student test) or ${ }^{b}$ median and interquartile range (Wilcoxon test); BMC - bone mineral content; BMD bone mineral density 
Table 3. Serum concentrations of biochemical bone metabolism markers in bone tumor survivors compared with healthy children

\begin{tabular}{|c|c|c|c|}
\hline & Bone tumor survivors & Healthy children & $\mathrm{p}$ \\
\hline Calcium $(\mathrm{mmol} / \mathrm{L})^{\mathrm{a}}$ & $2.34 \pm 0.10$ & $2.40 \pm 0.08$ & 0.033 \\
\hline Phosphate $(\mathrm{mmol} / \mathrm{L})^{\mathrm{a}}$ & $1.21 \pm 0.24$ & $1.34 \pm 0.22$ & 0.041 \\
\hline 25-hydroxyvitamin $\mathrm{D}(\mathrm{ng} / \mathrm{mL})^{\mathrm{a}}$ & $13.3 \pm 7.4$ & $22.5 \pm 6.5$ & $<0.001$ \\
\hline $\operatorname{ALP}(\mathrm{U} / \mathrm{L})^{\mathrm{b}}$ & $172.7(110.0-268.0)$ & $178.0(93.5-238.0)$ & 0.151 \\
\hline $\operatorname{BALP}(\mathrm{U} / \mathrm{L})^{\mathrm{b}}$ & $78.9(54.3-143.0)$ & $98.5(54.6-131.7)$ & 0.501 \\
\hline $\mathrm{OC}(\mathrm{ng} / \mathrm{mL})^{\mathrm{a}}$ & $74.4 \pm 26.7$ & $75.9 \pm 38.2$ & 0.800 \\
\hline $\operatorname{cOC}(\mathrm{ng} / \mathrm{mL})^{\mathrm{a}}$ & $18.7 \pm 10.4$ & $26.0 \pm 11.4$ & 0.008 \\
\hline $\mathrm{ucOC}(\mathrm{ng} / \mathrm{mL})^{\mathrm{a}}$ & $38.3 \pm 16.2$ & $29.7 \pm 14.9$ & 0.022 \\
\hline Ratio $\mathrm{cOC} / \mathrm{ucOC}^{\mathrm{b}}$ & $0.49(0.25-0.67)$ & $0.83(0.58-1.51)$ & 0.003 \\
\hline $\mathrm{CTX}(\mathrm{ng} / \mathrm{mL})^{\mathrm{a}}$ & $1.901 \pm 0.999$ & $1.669 \pm 0.874$ & 0.297 \\
\hline Ratio RANKL/OPG ${ }^{\mathrm{b}}$ & $2.64(0.85-17.38)$ & $3.18(1.34-10.82)$ & 0.954 \\
\hline
\end{tabular}

Data are presented as a mean values \pm SD (paired t-Student test) or ${ }^{b}$ median and interquartile range (Wilcoxon test); ALP - alkaline phophatase; BALP - bone alkaline phophatase; OC - osteocalcin; COC - carboxylated form of osteocalcin; ucOC - undercarboxylated form of osteocalcin; CTX - carboxyterminal telopeptide of collagen type I; OPG - osteoprotegerin; RANKL - receptor activator of nuclear factor kappaB ligand

$(\mathrm{p}<0.05)$ in patients with bone cancer than in the controls. Hence, the ratio of $\mathrm{cOC} / \mathrm{ucOC}$ was lower in patients in comparison with the control group $(\mathrm{p}<0.01)$. Additionally, we observed slightly higher concentrations of bone resorption markers (CTX) and lower ratio of RANKL/OPG in patients affected by bone tumors than in healthy children, but these differences were not statistically significant. We observed strong positive correlations between activity of BALP and concentrations of OC (Spearman 's rho 0.744, $\mathrm{p}<0.001$ ) as well as between BALP and CTX (Spearman`s rho 0.466, $\mathrm{p}=0.014$ ) in the patient group. No correlations between other serum bone metabolism markers were found.

Additionally, we presented differences $(\Delta)$ in anthropometric and biochemical parameters between patients and matched controls in two subgroups of patients (treated chemotherapy with MTX and without MTX) (Table 4). We found no sig-

Table 4. The differences in anthropometric and biochemical parameters between bone tumor survivors (treated with MTX and without MTX) and matched healthy subjects

\begin{tabular}{|c|c|c|c|}
\hline & \multicolumn{2}{|c|}{ Difference between patients with bone tumors and matched healthy subjects $(\Delta)$} & \multirow{2}{*}{$\mathrm{p}$} \\
\hline & treated with MTX & treated without MTX & \\
\hline Age (years) & $0.50(-0.50-2.50)$ & $0.00(-2.00-2.00)$ & 0.175 \\
\hline Height $(\mathrm{cm})$ & $-6.75(-19.00-22.00)$ & $-4.25(-21.00-20.00)$ & 0.525 \\
\hline Weight $(\mathrm{kg})$ & $-4.50(-16.00-58.00)$ & $2.00(-12.00-16.00)$ & 0.161 \\
\hline Body mass index $\left(\mathrm{kg} / \mathrm{m}^{2}\right)$ & $-0.04(-3.76-19.93)$ & $2.16((-3.79-5.98)$ & 0.262 \\
\hline Total fat mass $(\mathrm{kg})$ & $1.55(-5.96-36.26)$ & $6.99(-3.05-11.75)$ & 0.212 \\
\hline Total lean mass (kg) & $-6.22(-17.31-18.76)$ & $-5.47(-15.57-6.93)$ & 0.570 \\
\hline Total body BMD $\left(\mathrm{g} / \mathrm{cm}^{2}\right)$ & $-0.03(-0.25-0.08)$ & $-0.05(-0.34-0.17)$ & 0.920 \\
\hline Lumbar spine BMDL2-L4 $\left(\mathrm{g} / \mathrm{cm}^{2}\right)$ & $-0.09(-0.24-0.17)$ & $-0.05(-0.35-0.22)$ & 0.920 \\
\hline Total body BMD Z-score & $-0.85(-3.80-0.80)$ & $-0.90(-5.40-0.20)$ & 0.802 \\
\hline Lumbar spine BMDL2-L4 Z-score & $-1.30(-2.40-0.20)$ & $-0.75(-4.50-1.00)$ & 0.431 \\
\hline Calcium $(\mathrm{mmol} / \mathrm{L})$ & $-0.03(-0.16-0.20)$ & $-0.07(-0.40-0.11)$ & 0.340 \\
\hline Phosphate (mmol/L) & $-0.15(-0.58-0.43)$ & $0.05(-0.86-0.30)$ & 0.578 \\
\hline 25-hydroxyvitamin $\mathrm{D}$ (ng/mL) & $-5.10(-22.00-7.10)$ & $-10.80(-30.00-6.50)$ & 0.035 \\
\hline Bone alkaline phosphatase (U/L) & $-20.05(-94.70-58.10)$ & $5.50(-112.20-176.70)$ & 0.758 \\
\hline Osteocalcin (ng/mL) & $-3.15(-59.20-22.70)$ & $1.40(-52.80-67.60)$ & 0.537 \\
\hline Ratio cOC/ucOC & $-0.04(-1.34-0.68)$ & $-0.55(-3.67-0.32)$ & 0.066 \\
\hline CTX (ng/mL) & $-0.49(-2.03-1.42)$ & $0.44(-0.83-3.18)$ & 0.031 \\
\hline Ratio RANKL/OPG & $56.25(-12.80-80.37)$ & $35.34(-35.65-102.16)$ & 0.482 \\
\hline
\end{tabular}

Data are presented as medians of differences $(\Delta)$ between patients with bone tumors and matched healthy subjects and ranges; Mann-Whitney exact test was used for comparisons between differences in the MTX (methotrexate) group and the non-MTX group (minimum, maximum). 
nificant differences in $\Delta$ regarding body height, body weight, BMI, fat mass and lean mass, BMC and BMDs between MTX and non-MTX patients. Besides, we observed significantly $(\mathrm{p}<0.05)$ lower $\Delta$ in concentration of 25 -hydroxyvitamin $\mathrm{D}$ and higher $\Delta$ in concentration of CTX in patients treated with chemotherapy without MTX than in patients treated with MTX. We also noticed that $\Delta$ in the ratio of cOC/ucOC trended to be lower in the subgroup of patients that received chemotherapy without MTX ( $\mathrm{p}=0.066)$.

\section{Discussion}

In this study, we presented the results of anthropometric and biochemical bone metabolism parameters in children and adolescents with bone tumors just after the completion of therapy in comparison with healthy controls. We observed significantly lower values of $\mathrm{BMC}$ and $\mathrm{BMD}$ in the patient group than in the controls. About $30-35 \%$ of our patients had $\mathrm{z}$-score BMDs (total and lumbar spine) of -1 to -2 and about $20 \%$ had $z$-score BMDs less than -2 . There is limited information on bone mineral density in children and adolescents affected by malignant bone tumors. Only Muller et al [16] reported that $30 \%$ of pediatric bone sarcoma patients had decreased lumbar bone mineral density (z-score BMD <-1 $\mathrm{SD})$ even after preoperative chemotherapy. Most of the existing literature data on bone mineral density in bone sarcoma patients focused on adults and reported reduced BMD values and high prevalence of osteoporosis in long-term survivors $[3,15,17]$. Similar to us, decreases in total and regional BMDs in young adults with bone sarcomas after anti-cancer therapy were observed by Holzer et al [4], Ruza et al [5], and PirkerFruhauf et al [12].

Osteoporosis has usually been considered a disease that affects the elderly, but researchers have proved that it has pediatric origins [15,18-19]. Lim et al (16) observed that bone tumor survivors diagnosed at a young age showed a higher prevalence of osteoporosis than survivors diagnosed at an older age (37.5\% vs. $10.0 \%)$. These authors suggested that even values of $\mathrm{z}$-score BMD below - 1 increase the risk of fractures 2.5 -fold and have important repercussions on future quality of life. A statistically significant association between lean mass and bone mineral density in cancer survivors had been previously reported. In the present study, we found significantly lower lean mass and higher fat mass in the patient group than in the controls. We also observed strong positive correlation between body composition parameters and BMDs (total BMD and lumbar spine BMD) as well as and BMC in patients. Similarly to us, other authors reported alterations in lean mass and its relation with BMD in osteosarcoma adult survivors $[15,20]$.

Apart from assessments of anthropometric parameters by DXA, we analyzed also biochemical markers of bone metabolism which detect alterations over short time periods and may give information on the mechanisms associated with these disturbances. We observed comparable values of bone formation
(BALP, OC) and resorption (CTX) markers in children and adolescents with bone tumors and in age- and sex-matched controls. It has been recently shown that bone remodeling depends on the balance of cytokines which are predominantly secreted by osteoblasts. Among them, RANKL promotes the differentiation of osteoclasts precursors and the activation of resorption, whereas OPG inhibits the action of RANKL. An imbalance in the RANKL/OPG ratio leads to higher resorption, decreased BMD, and finally to osteoporosis [21-22]. In the present study, we observed a similar RANKL/OPG ratio in bone cancer survivors and the controls.

To our knowledge, are only few data on bone markers relating to children and adolescents with bone sarcomas after anticancer treatment. Only a few studies reported levels of biochemical bone markers in adult bone tumor survivors. Holzer et al [4] observed normal serum bone formation markers (BALP, OC) but elevated level of resorption marker (CTX) in osteosarcoma patients (ten years after completion of treatment). The authors speculated that for their patients lower BMD is related to bone resorption due to increased osteoclast activity. In another study, lower levels of bone formation markers (carboxyterminal propeptide procollagen type I - PICP, OC) and increased (in about 20\%) resorption marker (carboxyterminal cross-linked telopeptide of type I collagen - ICTP) in bone cancer survivors were found [5]. Pirker-Fruhauf et al [12] showed increased PICP, BALP, OC, CTX, TRAP (tartrate-resistant acid phosphatase) levels, and decreased RANKL concentration in some patients with bone cancers. However, these differences were not statistically significant. The above studies were conducted on adult patients, and the results were compared with reference data not a control group.

Apart from measurement of total osteocalcin concentration, assessment of its carboxylated and undercalboxylated forms are also very important. The posttranslational $\gamma$-carboxylation of osteocalcin causes the formation of the carboxylated form of this protein with greater affinity for calcium than undercarboxylated osteocalcin. Recent data demonstrated that in adults a higher concentration of ucOC, as a consequence of vitamin $\mathrm{K}$ insufficiency, is associated with low BMD and increased risk of osteoporotic fractures [23-24]. Little is known about levels of osteocalcin forms in growing bone with high metabolic activity. Van Summeren et al [26] reported that $\mathrm{COC}$ and ucOC in healthy children and adolescents were higher than in adults. Additionally, both forms of OC positively correlated with other markers of bone turnover but not with vitamin $\mathrm{D}$. We reported for the first time that in patients with malignant bone tumors after treatment, the concentrations of carboxylated osteocalcin were significantly lower and undercarboxylated OC higher than in the controls. Hence, the ratio of $\mathrm{cOC} / \mathrm{ucOC}$ was lower in patients affected by bone tumors than in healthy children and this might suggest alteration in bone formation and the mineralization processes. Therefore, alterations in bone turnover markers may lead to inferior bone quality or suboptimal bone mineralization resulting in increased fracture risk. 
Osteopathy due to chemotherapy is a well-known secondary effect. One of the important factors with a negative influence on BMD are high-doses of methotrexate. On the other hand, low-dose MTX treatment, used on patients with rheumatoid arthritis, does not seem to affect bone metabolism, even in the long term [26]. In our preliminary study, we did not observe statistically significant differences in anthropometric parameters in the subgroups of patients treated with chemotherapy with and without MTX. With regard to biochemical bone markers, we found significantly lower concentrations of vitamin $\mathrm{D}$ and higher CTX levels, as well as a trend of a lower ratio of $\mathrm{cOC} / \mathrm{ucOC}$ in patients treated without MTX than in those treated with MTX. Decreased levels of vitamin D and carboxylated form of osteocalcin together with elevated CTX concentrations might suggest an imbalance between bone formation and resorption processes. Most probably longer observation is required to achieve decrements in bone mineral density. We suggest that bone status after completion of chemotherapy was not affected by MTX only, but also by other factors, including treatment duration. It is not excluded that longer duration of therapy as well as radiotherapy which was used in $30 \%$ of patients with Ewing 's sarcoma negatively affected bone status. However, the small number of subjects included in the current study precludes a definite conclusion and needs confirmation in a larger group of patients.

Many cancer survivors have the additional risk factors of nutritional deficiency that result in altered calcium, phosphate and vitamin D metabolism [27]. It is commonly known, that during the pubertal years, mineral and vitamin sufficiency is important for optimal calcium absorption and bone mass acquisition [28]. Vitamin D plays an important role in the development, differentiation and mineralization of osteoblasts and, as shown recently, also in osteoclasts activity and bone resorption [29-30]. According to actual guidelines 25hydroxyvitamin $\mathrm{D}$ concentrations below $20 \mathrm{ng} / \mathrm{ml}$ indicate vitamin D deficiency, concentrations of $20 \mathrm{ng} / \mathrm{ml}$ up to $30 \mathrm{ng} /$ $\mathrm{ml}$ reflect a suboptimal status, and concentrations higher than $30 \mathrm{ng} / \mathrm{ml}$ up to $50 \mathrm{ng} / \mathrm{ml}$ reflect adequate status of this vitamin [31]. Several studies documented a high prevalence of vitamin $\mathrm{D}$ deficiency in cancer patients $[5,12]$. The observed vitamin $\mathrm{D}$ deficiency in $78 \%$ and its suboptimal status in $20 \%$ of our bone cancer survivors along with low calcium levels may affect bone turnover, mineralization and loss of BMD. We speculate that our patients were also insufficient in vitamin $\mathrm{K}$, because of increased levels of ucOC. Therefore, mineral and vitamin supplementation may be a possible strategy in the prevention of osteoporosis in childhood cancer survivors.

Our study had several limitations. First, we had a relatively small number of participants owing to the rarity of malignant bone tumor, the mortality rate, and inclusion criteria concerning adolescence period. Despite that, we can report a significant reduction in BMD (total and lumbar spine) and alterations in bone metabolism markers with potential decrease in bone formation in young patients with bone sarcomas. Secondly, we investigated patients shortly after the completion of chemotherapy. However, we are planning to conduct observations of the studied patients and repeat densitometry and biochemical analyses in the future. Thirdly, with a small number of patients in the subgroups (treated with and without MTX), it is not easy to draw a solid conclusion, but these are only preliminary results. The subgroups of patients treated without MTX consisted not only of patients with Ewing's sarcoma but also with PNET, however, these patients received similar chemotherapy. Finally, we did not examine dietary nutrient intake in the studied patients, but we measured serum 25-hydroxyvitamin D levels, a marker of vitamin $\mathrm{D}$ adequacy.

In summary, childhood and adolescence represent a critical time to attain peak bone mass. Treatment for bone sarcomas may interfere with the accumulation of an appropriate peak bone mass and may lead to an increased risk of osteoporosis in adulthood, causing reduction in the quality of life. Therefore, determination of BMD and biochemical bone metabolism markers in long-term survivors after chemotherapy for malignant bone tumors is important to determine patients at high risk of developing osteoporosis in their life.

Acknowledgments: The authors would like to thank the staff of the Department of Internal Medicine, Endocrinology and Diabetology at the Central Clinical Hospital MSW in Warsaw for measuring BMD by DXA.

\section{References}

[1] BACCI G, LONGHI A, VERSARI M, MERCURI M, BRICCOLI A et al. Prognostic factors for osteosarcoma of the extremity treated with neoadjuvant chemotherapy. Cancer 2006; 106: 1154-1161. http://dx.doi.org/10.1002/cncr.21724

[2] AKIYAMA T, DASS CR, CHOONG PFM. Novel therapeutic strategy for osteosarcoma targeting osteoclast differentiation, bone-resorbing activity, and apoptosis pathway. Mol Cancer Ther 2008; 7: 3461-3469. http://dx.doi.org/10.1158/15357163.MCT-08-0530

[3] KASTE SC, AHN H, LIU T, LIU W, KRASIN MJ et al. Bone mineral density deficits in patients treated for sarcoma. Pediatr Blood Cancer 2008; 50: 1032-1038. http://dx.doi.org/10.1002/ pbc. 21281

[4] HOLZER G, KREPLER P, KOSCHAT MA, GRAMPP S, DOMINKUS $\mathrm{M}$ et al. Bone mineral density in long-term survivors of highly malignant osteosarcoma. J Bone Joint Surgery 2003; 85: 231-237. http://dx.doi.org/10.1302/0301620X.85B2.13257

[5] RUZA E, SIERRASESUMAGA L, AZCONA C, PATINO-GARCIA A. Bone mineral density and bone metabolism in children treated for bone sarcomas. Pediatr Res 2006; 59: 866-871. http:// dx.doi.org/10.1203/01.pdr.0000219129.12960.c2

[6] MUSZYNSKA-ROSLAN K, KONSTANTYNOWICZ J, KRAWCZUK-RYBAK M, KACZMARSKI M, PIOTROWSKA-JASTRZEBSKA J. Bone mineral density in children before and after completion of cancer treatment. Pol Merkur Lekarski 2004; 17: 37-42. 
[7] WASILEWSKI-MASKER K, KASTE SC, HUDSON MM, ESIASHVILI N, MATTANO LA et al. Bone mineral density deficits in survivors of childhood cancer: Long-term follow-up guidelines and review of the literature. Pediatrics 2008; 121: e705-713. http://dx.doi.org/10.1542/peds.2007-1396

[8] MINAUR NJ, JEFFERISS C, BHALLA AK, BERESFORD JN. Methotrexate in the treatment of rheumatoid arthritis. I. In vitro effects on cells of the osteoblast lineage. Rheumatology (Oxford) 2002; 41: 735-740. http://dx.doi.org/10.1093/ rheumatology/41.7.735

[9] MINAUR NJ, KOUNALI D, VEDI S, COMPSTON JE, BERESFORD JN et al. Methotrexate in the treatment of rheumatoid arthritis. II. In vivo effects on bone mineral density. Rheumatology (Oxford) 2002; 41: 741-749. http:// dx.doi.org/10.1093/rheumatology/41.7.741

[10] UEHARA R, SUZUKI Y, ICHIKAWA Y. Methotrexate (MTX) inhibits osteoblastic differentiation in vitro: possible mechanism of MTX osteopathy. J Rheumatol 2001; 28: 251-256.

[11] FAN C, GEORGIOU KR, KING TJ, XIAN CJ. Methotrexate toxicity in growing long bones of young rats: a model for studying cancer chemotherapy-induced bone growth defects in children. J Biomed Biotechnol 2011; doi:10.1155/2011/903097. http://dx.doi.org/10.1155/2011/903097

[12] PIRKER-FRUHAUF UM, FRIESENBICHLER J, E-C URBAN, OBERMAYER-PIETSCH B, LEITHNER A. Osteoporosis in children and young adults: a late effect after chemotherapy for bone sarcoma. Clin Orthop Relat Res 2012; 470: 2874-2885. http://dx.doi.org/10.1007/s11999-012-2448-7

[13] LEE JA, JUNG JS, KIM DH, LIM JS, KIM MS et al. RANKL expression is related to treatment outcome of patients with localized, high-grade osteosarcoma. Pediatr Blood Cancer 2011; 56: 738-743. http://dx.doi.org/10.1002/pbc.22720

[14] COSTA-RODRIGUES J, TEIXEIRA CA, FERNANDES MH. Paracrine-mediated osteoclastogenesis by the osteosarcoma MG63 cell line: is RANKL/RANK signaling really important? Clin Exp Metastasis 2011; 28: 505-514. http://dx.doi. org/10.1007/s10585-011-9387-7

[15] LIM JS, KIM DH, LEE JA, KIM DH, CHO J et al. Young age at diagnosis, male sex, and decreased lean mass are risk factors of osteoporosis in long-term survivors of osteosarcoma. J Pediatr Hematol Oncol 2013; 35: 54-60. http://dx.doi.org/10.1097/ MPH.0b013e318275193b

[16] MUller C, WinTER CC, ROSENBAUM D, BOOS J, GOSHEGER G et al. Early decrements in bone density after completion of neoadjuvant chemotherapy in pediatric sarcoma patients. BMC Musculoskelet Disord 2010; 11: 287. doi:10.1186/1471-2474-11-287. http://dx.doi. org/10.1186/1471-2474-11-287

[17] HOFSTAETTER JG, ROSCHGER A, PUCHNER SE, DOMINKUS M, SULZBACHER I et al. Altered matrix mineralization in a case of a sclerosing osteosarcoma. Bone 2013; 53: 409-413. http://dx.doi.org/10.1016/j.bone.2012.12.043

[18] COX CL, HUDSON MM, MERTENS A, OEFFINGER K, WHITTON J et al. Medical screening participation in the childhood cancer survivor study. Arch Intern Med 2009; 169: 454-462. http://dx.doi.org/10.1001/archinternmed.2008.588
[19] AZCONA C, BURGHARD E, RUZA E, GIMENO J, SIERRASESUMAGA L. Reduced bone mineralization in adolescent survivors of malignant bone tumors: comparison of quantitative ultrasound and dual-energy x-ray absorptiometry. J Pediatr Hematol Oncol 2003; 25: 297-302. http://dx.doi. org/10.1097/00043426-200304000-00006

[20] POLGREEN LE, PETRYK A, DIETZ AC, SINAIKO AR, LEISENRING W et al. Modifiable risk factors associated with bone deficits in childhood cancer survivors. BMC Pediatr 2012; 12: 40. doi:10.1186/1471-2431-12-40. http://dx.doi. org/10.1186/1471-2431-12-40

[21] LAMOUREUX F, RICHARD P, WITTRANT Y. Therapeutic relevance of osteoprotegerin gene therapy in osteosarcoma: blockade of the vicious cycle between tumor cell proliferation and bone resorption. Cancer Res 2007; 67: 7308-7318. http:// dx.doi.org/10.1158/0008-5472.CAN-06-4130

[22] BOYCE BF, XING L. Functions of RANKL/RANK/OPG in bone modeling and remodeling. Arch Biochem Biophys 2008; 473: 139-146. http://dx.doi.org/10.1016/j.abb.2008.03.018

[23] GUNDBERG CM, LIAN JB, BOOTH SL. Vitamin K-dependent carboxylation of osteocalcin: friend or foe? Adv Nutr 2012; 3: 149-157. http://dx.doi.org/10.3945/an.112.001834

[24] NEVE A, CORRADO A, CANTATORE FP. Osteocalcin: skeletal and extra-skeletal effects. J Cell Physiol 2013; 228: 1149-1153. http://dx.doi.org/10.1002/jcp.24278

[25] VAN SUMMEREN MJH, VAN COEVERDEN SCCM, SCHYRGERS LJ, BRAAM LAJ, NOIRT F et al. Vitamin K status is associated with childhood bone mineral content. Br J Nutr 2008; 100: 852-858. http://dx.doi.org/10.1017/ $\underline{\text { S0007114508921760 }}$

[26] Revu S, Neregard P, Klint E, Korotkova M, CATRINA AI. Synovial membrane immunohistology in early-untreated rheumatoid arthritis reveals high expression of catabolic bone markers that is modulated by methotrexate. Arthritis Res Therapy 2013; 15: R2015. http://dx.doi.org/10.1186/ar4398

[27] CHOI YJ, PARK SY, CHO WK, LEE JW, CHO KS et al. Factors related to decreased bone mineral density in childhood cancer survivors. J Korean Med Sci 2013; 28: 1632-1638. http://dx.doi.org/10.3346/jkms.2013.28.11.1632

[28] TYLAVSKY FA, SMITH K, SURPRISE H, GARLAND S, YAN X et al. Nutritional intake of long-term survivors of childhood acute lymphoblastic leukemia: evidence for bone health interventional opportunities. Pediatr Blood Cancer 2010; 55: 1362-1369. http://dx.doi.org/10.1002/pbc.22737

[29] VASHI PG, TRUKOVA K, LAMMERSFELD CA, BRAUN DP, GUPTA D. Impact of oral vitamin D supplementation on serum 25-hydroxyvitamin D levels in oncology. Nutr J 2010; 9: 60. http://dx.doi.org/10.1186/1475-2891-9-60

[30] BASIT S. Vitamin D in health and disease: a literature review. Br J Biomed Sci 2013; 70: 161-172.

[31] PLUDOWSKI P, KARCZMAREWICZ E, BAYER M, CARTER G, CHLEBNA-SOKOL D et al. Practical guidelines for the supplementation of vitamin $\mathrm{D}$ and the treated of deficits in Central Europe - recommended vitamin D intakes in the general population and groups at risk of vitamin deficiency. Endokrynol Pol 2013; 64: 319-327. http://dx.doi.org/10.5603/ $\underline{\text { EP.2013.0012 }}$ 